\title{
Diversity and composition of the Panax ginseng rhizosphere microbiome in various cultivation modesand ages
}

\author{
Ai-Zi Tong ${ }^{1}$, Wei Liu", Qiang Liu², Guang-Qing Xia ${ }^{1}$ and Jun-Yi Zhu
}

\begin{abstract}
Background: Continuous cropping of ginseng (Panax ginseng Meyer) cultivated in farmland for an extended period gives rise to soil-borne disease. The change in soil microbial composition is a major cause of soil-borne diseases and an obstacle to continuous cropping. The impact of cultivation modes and ages on the diversity and composition of the $P$. ginseng rhizosphere microbial community and technology suitable for cropping $P$. ginseng in farmland are still being explored.

Methods: Amplicon sequencing of bacterial 165 rRNA genes and fungal ITS regions were analyzed for microbial community composition and diversity.

Results: The obtained sequencing data were reasonable for estimating soil microbial diversity. We observed significant variations in richness, diversity, and relative abundances of microbial taxa between farmland, deforestation field, and different cultivation years. The bacterial communities of LCK (forest soil where $P$. ginseng was not grown) had a much higher richness and diversity than those in NCK (farmland soil where $P$. ginseng was not grown). The increase in cultivation years of $P$. ginseng in farmland and deforestation field significantly changed the diversity of soil microbial communities. In addition, the accumulation of $P$. ginseng soil-borne pathogens (Monographella cucumerina, Ilyonectria mors-panacis, I. robusta, Fusarium solani, and Nectria ramulariae) varied with the cropping age of $P$. ginseng.

Conclusion: Soil microbial diversity and function were significantly poorer in farmland than in the deforestation field and were affected by $P$. ginseng planting years. The abundance of common soil-borne pathogens of $P$. ginseng increased with the cultivation age and led to an imbalance in the microbial community.
\end{abstract}

Keywords: Cultivation ages, Cultivation modes, Panax ginseng, Rhizosphere microbiome

\section{Introduction}

As a traditional Chinese herbal medicine, the perennial plant ginseng (Panax ginseng Meyer) is famous for its benefits to the immune system [1, 2], nervous system [3], cardiovascular system [4-6], and has an anti-cancer effect $[7,8]$. P. ginseng is mainly grown in Asia,

\footnotetext{
* Correspondence: Iwtong@163.com

'School of Life Science, Tonghua Normal University, No.950 Yu Cai Road, Dongchang District, Tonghua 134002, China

Full list of author information is available at the end of the article
}

particularly in China, Korea, and Russia [9]. China has a long history of $P$. ginseng cultivation. In particular, $P$. ginseng produced from the Changbai Mountain area in eastern Jilin Province in China is well-known for its high yield and top quality [10]. For the past 400 years, $P$. ginseng has been mainly cultivated in the deforestation field. However, this cultivation method was banned by the Chinese government because of its negative impact on forest resources and the ecological environment. Currently, the cultivation is mainly conducted in the

C C The Author(s). 2021 Open Access This article is licensed under a Creative Commons Attribution 4.0 International License, which permits use, sharing, adaptation, distribution and reproduction in any medium or format, as long as you give appropriate credit to the original author(s) and the source, provide a link to the Creative Commons licence, and indicate if changes were made. The images or other third party material in this article are included in the article's Creative Commons licence, unless indicated otherwise in a credit line to the material. If material is not included in the article's Creative Commons licence and your intended use is not permitted by statutory regulation or exceeds the permitted use, you will need to obtain permission directly from the copyright holder. To view a copy of this licence, visit http://creativecommons.org/licenses/by/4.0/ The Creative Commons Public Domain Dedication waiver (http://creativecommons.org/publicdomain/zero/1.0/) applies to the data made available in this article, unless otherwise stated in a credit line to the data. 
farmland, which has led to serious soil-borne diseases of $P$. ginseng. Consequently, the yield and quality of $P$. ginseng cannot be guaranteed, causing huge economic losses and impeding the healthy and sustainable development of the national ginseng industry.

Soil microorganisms are responsible for the decomposition and cycling of organic compounds [11]. They also influence above-ground ecosystems by contributing to plant nutrition and health [12], and soil structure and fertility [13]. Rhizospheric microorganisms buffer the effects of toxic compounds in the soil and soil-borne pathogens [14]. Therefore, the soil microbial community is an important biological indicator of soil functions [15, 16]. The structure and relative activity of microbial communities are often different across plant species, cultivation years, and plant developmental stages [17]. A few recent studies on the association between continuous cropping and microbial community revealed that continuous farming led to alterations in microbial community diversity of rhizospheric soils [18-20]. For instance, compared with traditional crops, the diversity and composition of soil microbiome changed in soil with continuously planted American ginseng [20]. A significant change was also observed in the microbial communities of the arable soil with continuously planted $P$. ginseng [21]. In addition, different planting ages of $P$. ginseng could also alter soil microbial community [22].

$P$. ginseng grows continuously in fixed plots for 4 to 5 years. The soil-borne diseases of $P$. ginseng become more serious with the plant ages, causing root rot and rusty root, and reducing the survival rate of $P$. ginseng. Soilborne diseases may be influenced by the changes in soil microbial communities [23]. Accordingly, a direct association was observed between the prevalence of $P$. ginseng soil-borne diseases in farmlands and the pathogen accumulation [16]. Understanding the diversity and composition of microbial communities in different types of soil, and the relationship between microorganisms and cultivation ages of their host plants, such as $P$. ginseng, could be helpful in the prevention and control of soil-borne diseases and increase productivity. While polymerase chain reaction denaturing gradient gel electrophoresis and random amplification of polymorphic DNA methods are used to analyze the microbial diversity of $P$. ginseng rhizosphere soil at different ages and cultivation modes $[22,24]$, most microbes are still difficult to be analyzed using these traditional methods. Compared with traditional methods of analysis, highthroughput sequencing technology is one of the most widely used tools for evaluating $P$. ginseng rhizospheric bacterial and fungal community structure. In this study, we carried out amplicon sequencing to analyze the diversity and structure of $P$. ginseng rhizospheric microbial communities from farmland and deforestation field soil during zero to $5 \mathrm{y}$ of $P$. ginseng cultivation. This study allowed us to evaluate the effects of the two land types and cultivation years on the microbial communities.

\section{Results}

\section{Amplicon sequencing and rarefaction curves}

To characterize the microbiome in $P$. ginseng rhizospheric soil at different cultivation ages under two land types, 36 samples were sequenced by Illumina MiSeq. The amplicon sequencing resulted in a total of 1,362,294 reads of bacterial 16S rRNA genes, and 1,355,565 reads of fungal ITS regions after quality trimming and assigning. The average lengths of the obtained bacterial and fungal sequence were $436 \mathrm{bp}$ and $263 \mathrm{bp}$, respectively. High-quality sequences with $97 \%$ sequence similarity were gathered into 4061 bacterial OTUs and 3102 fungal OTUs.

To construct rarefaction curves, the dataset was flattened according to the minimum number of sequence of the samples. The rarefaction curves were constructed based on the number of OTUs observed in the individual sample (Fig. 1). The rarefaction curves for evaluating the richness of each sample usually approached saturation, indicating that the sequencing data were reasonable for evaluating the microbial diversity of $P$. ginseng rhizosphere. The fungal rarefaction curves exhibited a higher degree of variation in shape compared to that of bacterial OTUs.

\section{Alpha diversity of bacterial and fungal communities}

To estimate differences in the alpha diversity, we trimmed-off the minimum number sequence from samples of the dataset. Pairwise hierarchical clustering using Bray-Curtis dissimilarity revealed that the sample NCK3 was distinctly separated from NCK1 and NCK2. Therefore, we removed the NCK3 data from analyzing the alpha diversity. The OTU Chao richness index, the Simpsoneven evenness index, and the $\mathrm{Q}$ statistics of species diversity (Qstat) were used to reflect the alpha diversity of microbial communities in $P$. ginseng rhizosphere (Fig. 2). First, the alpha diversity of the microbial community was evaluated in the farmland and deforestation field with no $P$. ginseng planting. As expected, the bacterial communities of the deforestation field (LCK, forest soil where $P$. ginseng was not grown) had a much higher richness and diversity than those of the farmland (NCK, farmland soil where $P$. ginseng was not grown) (Fig. 2a). OTU richness value of LCK $(2035.1 \pm 15.8)$ was higher than that of NCK $(1746.3 \pm 21.6)$ and separated from NCK $(P<0.001)$. On the contrary, the LCK had a lower OTU evenness than that of NCK $(P<0.01)$. However, NCK and LCK did not exhibit variations in the richness, evenness, and diversity of the fungal communities (Fig. S1A). 

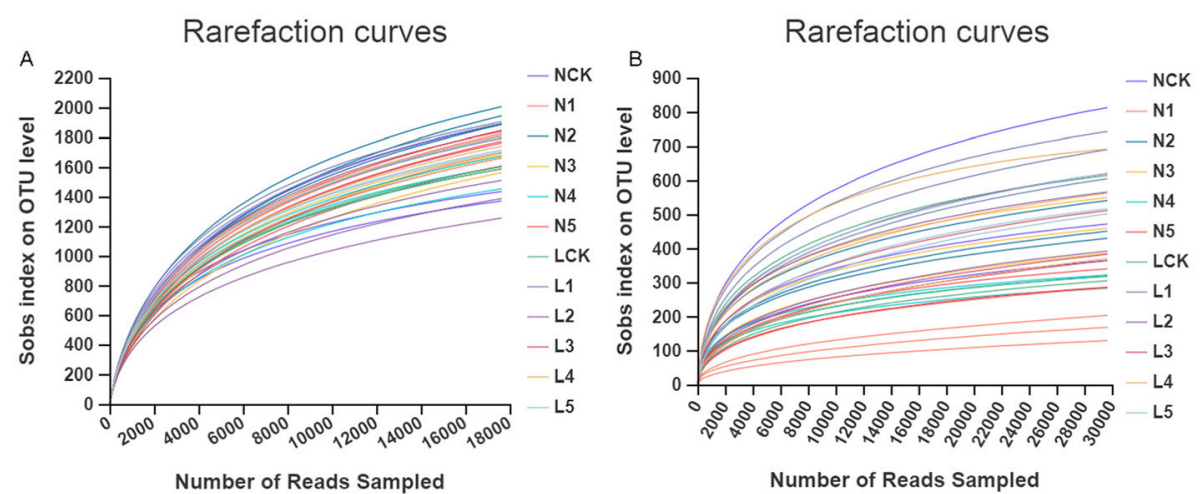

Fig. 1 Rarefaction curves of individual soil samples (a. bacteria, b. fungi).The rarefaction curve was assembled using the Sobs index at the OTU level. Relative to the total number of sequences, sequence similarity was defined at $97 \%$ cut-off by Mothur

P. ginseng is a perennial herb and that needs to grow continuously in the same place for many years. We observed that the alpha diversity of the soil microbial community changed significantly with the cultivation years of $P$. ginseng in farmland and deforestation field. The farmland and the deforestation field exhibited similar patterns of soil bacterial community richness and diversity in terms of the cultivation years (Fig. 2b, d). The richness and diversity of farmland soil bacteria showed an increasing trend in the first 2 y of $P$. ginseng planting and significantly decreased in the third year. However, in the first $3 \mathrm{y}$ of planting $P$. ginseng in the forest field, the bacterial richness and diversity first increased, then decreased, and increased again. In the fourth and fifth years of $P$. ginseng cultivation in both farm land and deforestation field, the bacterial richness and diversity were stable relative to those in the third year. To sum up, after growing $P$. ginseng continuously for five years (N5/ L5), the richness and diversity of soil bacteria increased compare to those in NCK or LCK. After P. ginseng cultivation in the farmland, fungal richness and diversity decreased in the first year, increased in the second and third years, and decreased again in the fourth and fifth years. The richness of the fungal community decreased in farmland soil after growing $P$. ginseng for five years (N5) compared to that in NCK, although the diversity did not change significantly (Fig. 2c). The fungal community richness and diversity in forest soil were less affected by the number of cultivation years and showed no marked variation in the richness and diversity after growing $P$. ginseng for five years (L5), compared with those in LCK (Fig. S1B).

\section{Beta diversity of bacterial and fungal communities}

We estimated beta diversity based on phylogenetic levels of OTUs of bacterial and fungal communities. The community composition in different samples was compared using the Bray-Curtis distance matrix and the primary factors driving the community composition were determined by calculating the abundance of reads after normalization and square root conversion. Hierarchical clustering was constructed based on Bray-Curtis dissimilarities. Principal coordinate analysis ( $\mathrm{PCoA}$ ) was performed to determine the overall similarity of microbial community structure among samples; when combined with the PERMANOVA analysis, it showed a significantly different community composition of bacteria $\left(\mathrm{R}^{2}=0.711, p=0.001\right)$ and fungi $\left(R^{2}=0.562, \quad p=0.001\right)$ in different sample groups. The farmland soil samples, except for NCK3, were clearly distinct from those of deforestation field, farmland, and forest soil samples (Fig. 3).

The hierarchical clustering of pairwise Bray-Curtis dissimilarities of bacterial OTUs revealed complete clustering according to the rhizosphere soil indifferent fields but did not cluster completely according to their cultivation years (Fig. 3a). PCoA analysis revealed that the horizontal coordinate was the primary coordinate component that caused differences in the composition of microbial community indifferent soil samples. In terms of the OTUs, the variance contribution rates of the PC1 to the difference in bacterial and fungal community composition of samples were 35.95 and $18.89 \%$, respectively (Fig. 3b, d).

\section{The composition and structure of the bacterial community}

From bacterial 16S rRNA gene sequences, 36 prokaryoticphyla, 89 classes, 180 orders, 346 families, 656 genera, 1439 species, and 4056OTUs were identified based on 97\% species similarity. The bacterial phyla Proteobacteria, Actinobacteria, Acidobacteria, and Chloroflexi were the richest in both the farmland or deforestation field under $P$. ginseng cultivation (Fig. 4a). We analyzed the dominant bacterial phyla and observed distinct 

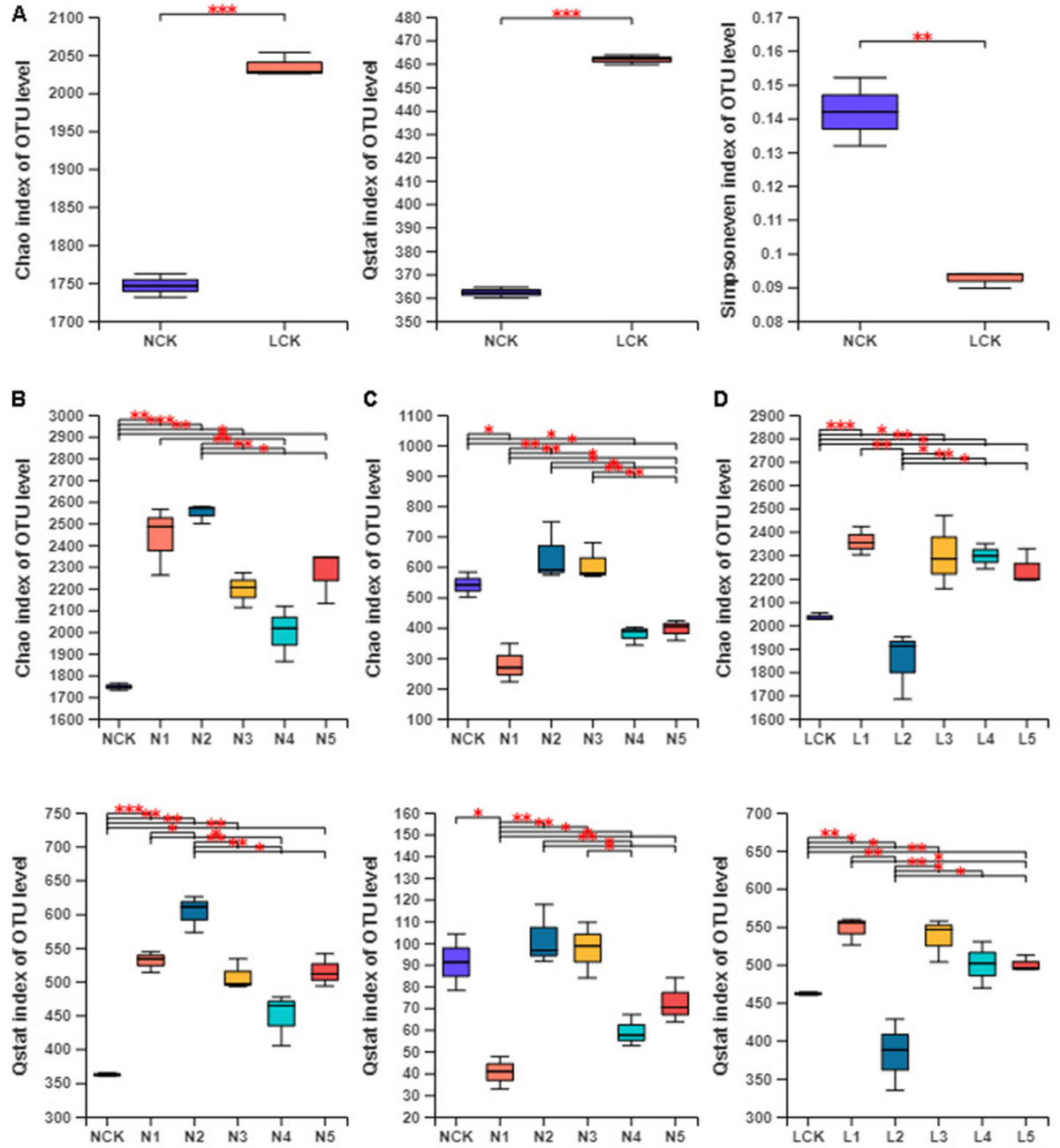

Fig. 2 Estimate the microbial community by alpha diversity. a, Alpha diversity of bacterial communities between NCK and LCK. b, Alpha diversity of bacterial communities among NCK, N1, N2, N3, N4, and N5. c, Alpha diversity of fungal communities among NCK, N1, N2, N3, N4, and N5. d, Alpha diversity of bacterial communities among $L C K, L 1, L 2, L 3, L 4$, and $L 5$. Estimation of Alpha diversity representing two biological replicates for the NCK samples, and three biological replicates for the other rhizospheric soil samples $\left({ }^{*} p<0.05,{ }^{* *} p<0.01,{ }^{* * *} p<0.001\right)$

bacterial communities in the two types of lands. Continuous cultivation years of $P$. ginseng in the same field also drastically affected the soil microbial community structure. The first 15 phyla were assessed using ANOVA to test the impact of different lands (NCK vs LCK, N5 vs L5) and cultivation years (NCK vs N5, LCK vs L5) on their relative abundance (\%) (Fig. 4 B). With the exception of Proteobacteria $(P=0.274)$, Cyanobacteria $(P=0.068)$, and Planctomycetes $(P=0.400)$, the top 15 relatively abundant bacterial phyla were significantly different among different samples (Fig. S2). In the LCK soil samples, we found significant enrichment $(P<0.05)$ of Verrucomicrobia, Nitrospirae, Latescibacteria, and Tectomicrobia (relative abundance $=7.64,6.48,0.75$, and $0.42 \%)$, compared to those in the NCK. The significant difference between the forest field (the L group) and the farmland (the $\mathrm{N}$ group) were further assessed by Student's t-test. The relative abundances of Actinobacteria (23.03\%), Verrucomicrobia (5.77\%), Nitrospirae (3.92\%), and Latescibacteria $(0.35 \%)$ were remarkably increased in the forest field as compared to those in the farmland, while Chloroflexi (9.70\%), Cyanobacteria (1.64\%), Bacteroidetes (2.27\%), Firmicutes (2.07\%), Gemmatimonadetes (1.35\%), Saccharibacteria (0.57\%), and Parcubacteria $(0.09 \%)$ were decreased in relative abundances. During the first year of $P$. ginseng cultivation in the farmland, the relative abundance of Acidobacteria (7.56\%) decreased compared to that in NCK (14.07\%). The relative abundance of Actinobacteria decreased in N2 (15.03\%) compare to N1 (25.98\%), while that of Parcubacteria increased in bothN1 and N2 (0.04 and $0.28 \%$, respectively). The relative abundance of Bacteroidetes in N1 (10.49\%) 

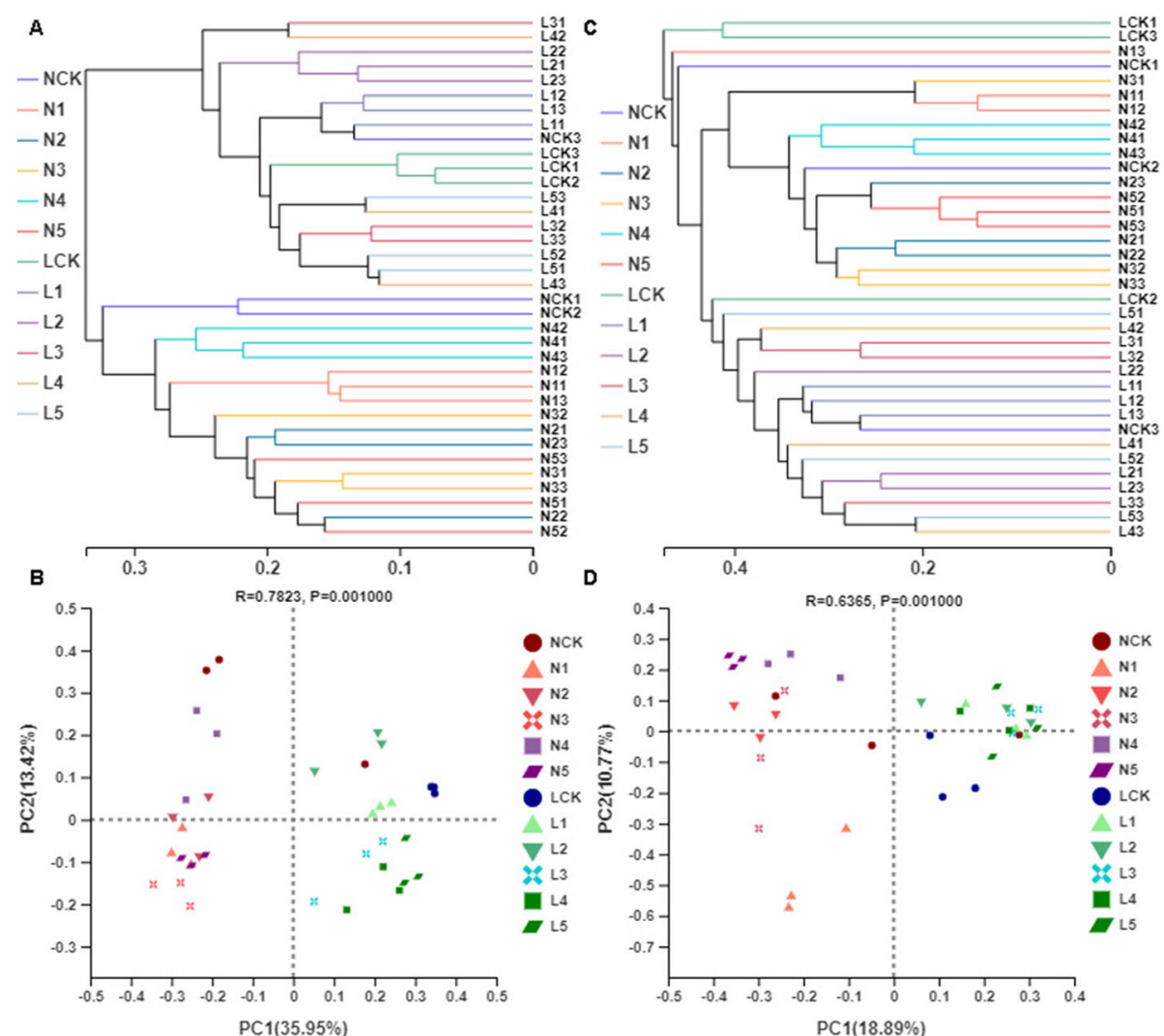

Fig. 3 Estimation of similarity and distance based on the hierarchical clustering tree of the samples (a, bacteria; c, fungi) and principal coordinate analysis (PCOA) (b, bacteria; d fungus) of the OTUs. The length of the branches in the hierarchical tree represents the distance between samples. The closer the two samples are on the PCoA plot, the more similar the species composition of the two samples

increased compared to that in NCK (1.41\%) and N2 (2.55\%). The relative abundance of Cyanobacteria decreased in N4 (0.81\%) compared to that in N3 (16.46\%), while that of Nitrospirae increased in both N3 and N4 ( 0.56 and $0.99 \%$, respectively). After five years of $P$. ginseng cultivation in farmland, the relative abundance of Acidobacteria (9.52\%) decreased significantly in N5 as compared to that in NCK, while the relative abundance of Bacteroidetes (2.62\%) increased (Fig. S3A).

In the first year of $P$. ginseng cultivation in the deforestation field, the relative abundance of Proteobacteria (LCK 29.75\%, L1 36.19\%), Actinobacteria (LCK 18.64\%, L1 24.41\%), and Saccharibacteria (LCK 0.16\%, L1 0.29\%) increased, while that of Chloroflexi (LCK 13.27\%, L1 9.02\%), Latescibacteria (LCK 0.75\%, L1 0.19\%), and Tectomicrobia (LCK $0.42 \%$, L1 $0.10 \%$ ) decreased. The relative abundance of Acidobacteria in L2 was less than that in L1 (9.54, and 14.95\%, respectively), while that of Saccharibacteria increased in both L1 and L2 (0.29, and $1.16 \%$, respectively). The relative abundance of Firmicutes in L3 was less than that in L2 (1.41 and 3.18\%, respectively). The relative abundance of Tectomicrobia was higher in L4 than that inL3 $(0.29$ and $0.14 \%$, respectively). After five years of continuous cultivation of $P$. ginseng in the forest field, there was a remarkable increase in the relative abundances of Actinobacteria (25.58\%), Bacteroidetes (2.14\%), and Cyanobacteria (1.11\%) in the L5 as compared with those in the LCK. Finally, the relative abundances of Actinobacteria (25.58\%), Nitrospirae (5.48\%), and Tectomicrobia $(0.38 \%)$ increased noticeably in L5 than inN5, while that of Gemmatimonadetes (1.41\%) decreased (Fig. S3B).

\section{The composition and structure of the fungal community}

From fungal ITS sequences,eighteukaryoticphyla,31 classes, 104 orders, 231 families,517 genera, 894species, and 3088 OTUs were identified. Fungal OTUs primarily consisted of phyla Ascomycota, Zygomycota, Basidiomycota, Rozellomycota, Chytridiomycota, and Glomeromycota. Ascomycota was the most abundant in the farmland as well as in the deforestation field during the cultivation of P. ginseng (Fig. 5a). We assessed the dominant fungal phyla using ANOVA, which distinguished the fungal communities in two different soil types and cultivation years based on their relative abundance (\%) (Fig. 5b).The relative abundance of Ascomycota and Zygomycota 


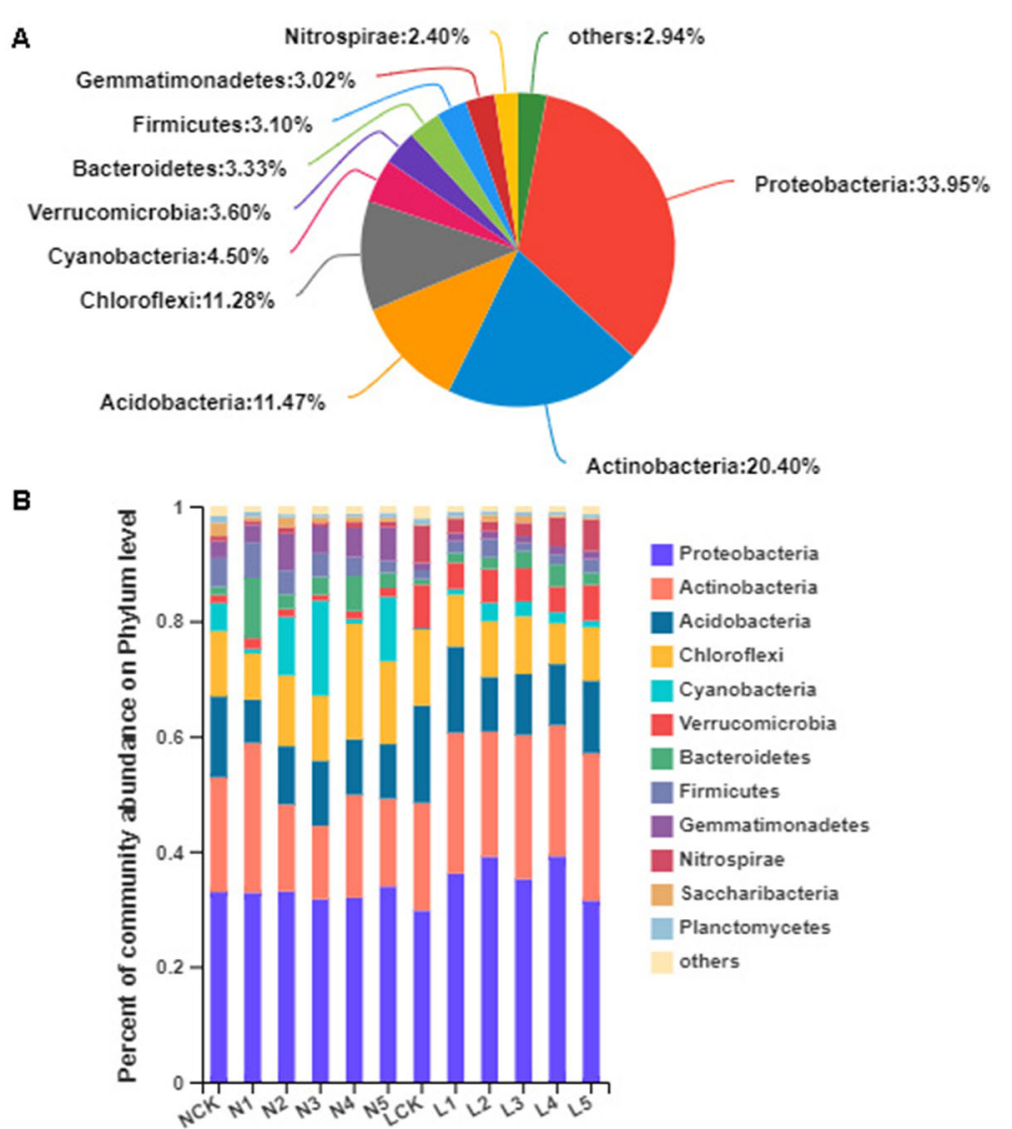

Fig. 4 The composition and structure of the bacterial community. A, Pieplot for bacterial community analysis at the phylum level. B, Relative abundances of the bacterial phyla in different samples

varied significantly $(P<0.05)$ on different soils and cultivation years among nearly all identified fungal phyla (Fig. S4). Compared to the NCK, no significant divergence of eukaryoticphyla was observed in the LCK samples. The student's t-test was performed to analyze any significant difference between the fungal communities in the forest field (group L) and the farmland (group $\mathrm{N})$. The relative abundances of Zygomycota and Glomeromycota increased significantly in the farmland (32.46and $0.46 \%$, respectively) as compared to those in the forest field (8.33and $0.03 \%$, respectively), while that of Basidiomycota (9.34and26.39\%, respectively) decreased (Fig. S5). In the second year of $P$. ginseng cultivation, there was an increase in the relative abundances of Glomeromycota and Rozellomycota in N2 (0.38 and $0.02 \%$, respectively) compared to those in the N1 soil sample, which was $0 \%$ for both phyla. The relative abundance of Rozellomycota in N3 (0\%) decreased compared to that in $\mathrm{N} 2(0.02 \%)$, and was nearly similarbetweenN3 and N4, and between $\mathrm{N} 4$ and N5. After growing P. ginseng for five years in the farmland, the relative abundance of Zygomycota (49.16\%) increased remarkably in N5 compared to that in NCK (Fig. S5). In different years of continuous $P$. ginseng plantation in the forest field, the variations in the relative abundances of eukaryoticphyla were relatively small. The relative abundance of Ascomycotain L1 increased remarkably (60.46\%) compared to that in LCK (32.71\%). The relative abundance of Zygomycota in L2 increased (15.53\%) compared to that in L1 (5.15\%) and L3 (3.96\%) and was nearly similar between L3 and L4, and between L4 and L5. After planting $P$. ginseng for five years in the forest field, the relative abundances of eukaryotic phyla did not vary significantly in L5 as compared to that in LCK. Finally, the relative abundance of Zygomycota was remarkably higher in N5 (49.16\%) than that in L5 (10.83\%) (Fig. S5).

\section{Pathogenic fungal enrichment varies with $P$. ginseng cropping age}

With the increase in the number of cultivation years of $P$. ginseng, its soil-borne diseases become progressively serious. Therefore, it was speculated that soil-borne pathogens accumulate with the increase of $P$. ginseng cropping years. Mortierella was the most abundant genus in the tested soil samples (Fig. 6a). Fusarium is a common pathogenic microorganism that causes soil- 


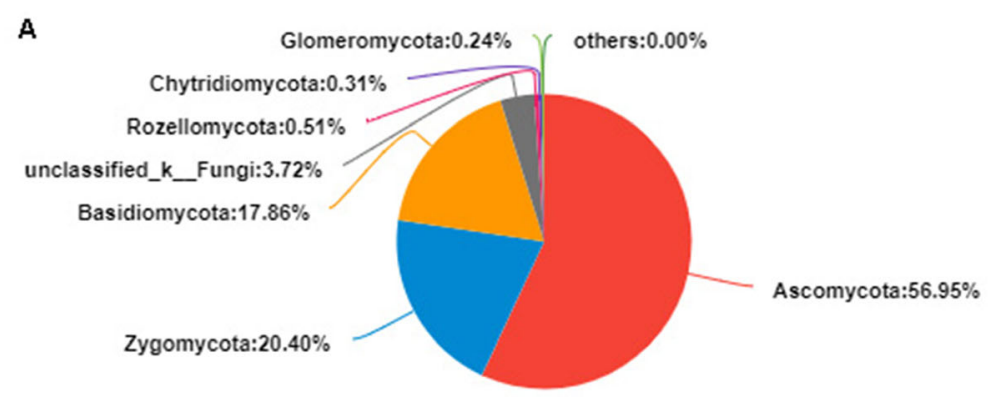

B

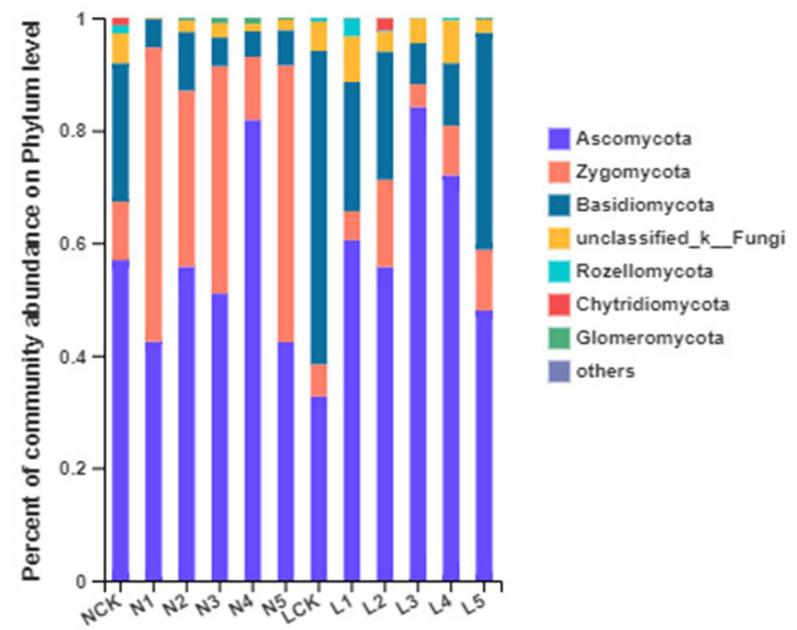

Fig. 5 The composition and structure of the fungal community. A, Pieplot for fungal community analysis at the phylum level. B, Relative abundances of the fungal phylain different samples

borne diseases in plants. In our study, Fusarium was estimated to be the third most abundant genus, which was much higher in farmland soil than in the forest soil. Gibberella, the perfect stage of Fusarium, was also detected in the samples [25]. In addition to ginseng root rot, another major $P$. ginseng soil-borne disease is ginseng rust rot, which is caused by Cylindrocarpon. We observed similar distribution trends of Cylindrocarpon and Nectria. Nectria belongs to Nectriaceae and is the perfect stage of Cylindrocarpon. With the cropping of $P$. ginseng, the abundances of Cylindrocarpon and Nectria increased and were the highest in L5 soil samples.

Accordingly, we further analyzed the changes in the abundances of pathogenic species. We found that five pathogenic species (Monographella cucumerina, Ilyonectria mors-panacis, I. robusta, Fusarium solani, and Nectria ramulariae) accumulated differently in $P$. ginseng rhizosphere soil samples of different plantation years and patterns (Fig. 6b). M. cucumerina significantly accumulated in the second year of $P$. ginseng planting and increased annually, reaching its maximum value in the fourth year in the farmland (8.14\%).The accumulation of M. cucumerina increased significantly in the third and fourth years of planting $P$. ginseng in the forest field (8.05 and $10.48 \%$,respectively).I. mors-panacis accumulated significantly in the fourth year of planting P. ginseng, in the farmland as well as in the forest field (18.84 and $4.23 \%$,respectively). I. robusta accumulated more in forest land than in farmland, especially from the second year of planting $P$. ginseng, and its accumulation increased annually, reaching the maximum in the fourth year (5.18\%). F. solani accumulated more in NCK $(0.59 \%)$ than in LCK $(0.09 \%)$, with the maximum found in the forest land with $P$. ginseng cultivation. $N$. ramulariae accumulated more in NCK $(0.29 \%)$ than in LCK $(0.08 \%)$, and its abundance decreased in the farmland with $P$. ginseng planting, but increased in the forest land, reaching the highest in the fifth year (1.45\%). In short, the accumulation of $P$. ginseng soil-borne pathogens varied with the cropping ages of $P$. ginseng as observed in our study.

\section{Discussion}

Soil-borne diseases are common and serious problems faced in the $P$. ginseng cultivation, especially in the farmlands. The soil fertility in farmlands with continuous 


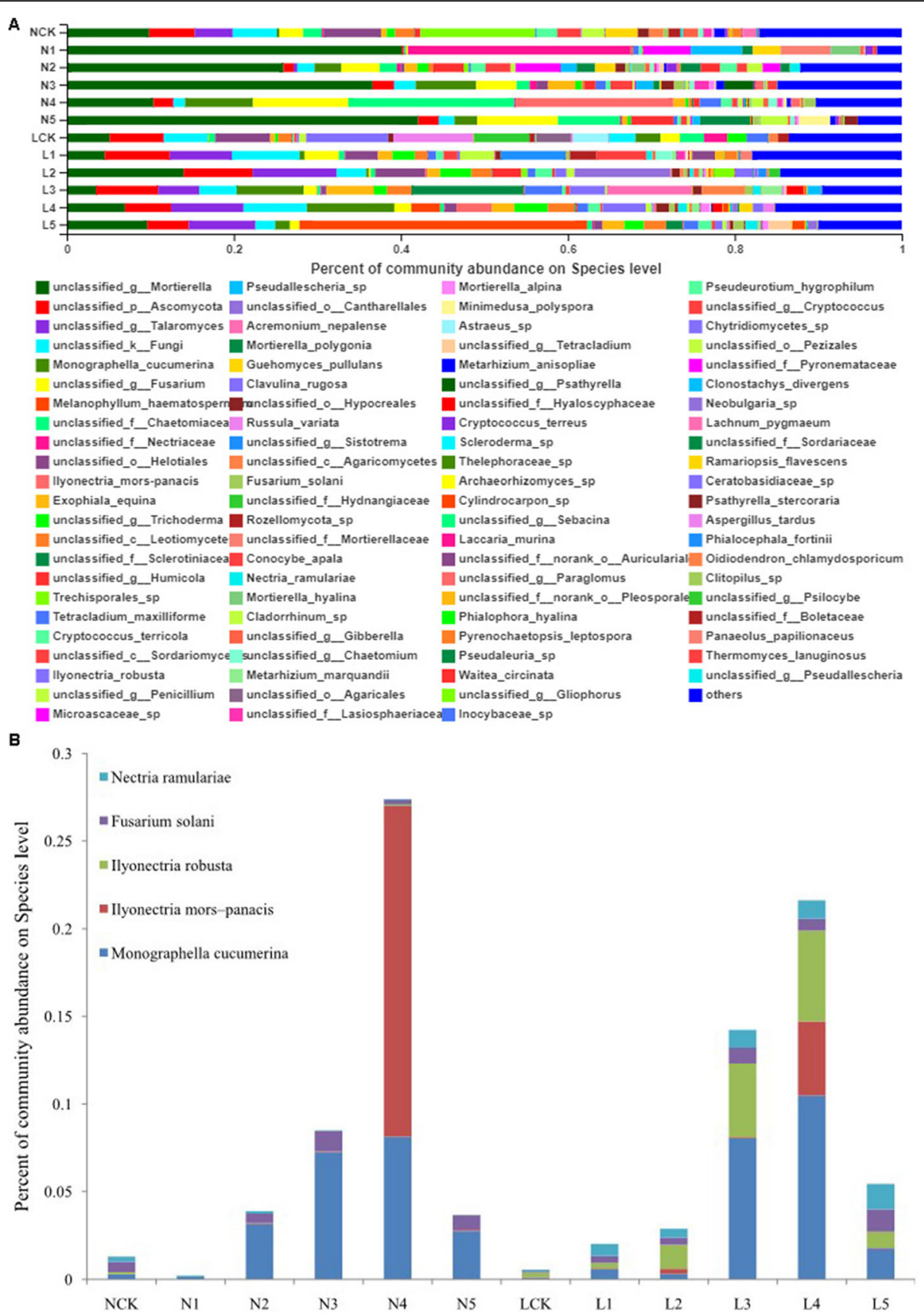

Fig. 6 The relative abundance of pathogenic fungal in soil samples. Three biological replicates of each sample. A, Relative abundances of the different fungal in different samples at genus level. B, Relative abundance of pathogenic fungal species in soil samples

planting of crops is maintained by increasing the use of chemical fertilizers, which reduces beneficial microorganisms and increases the content of soil-borne pathogens each year. The relationship between soil-borne diseases and soil microbiome under the continuous $P$. ginseng cultivation in farmlands seems to be important for epidemiological studies. In this study, we found that the $P$. ginseng cultivation, especially on different lands and for different durations, affects the diversity and structure of the microbial community in the $P$. ginseng rhizosphere, in accordance with findings from previous studies [24, 26]. Imbalances in rhizospheric microbial communities disrupt $P$. ginseng cultivation [24].

Soil microorganisms play a vital role in maintaining the stability and health of the soil ecological systems. Variations in the microbial community may adversely affect soil quality and plant health. Hence, it is important to study the variation in the soil microbial community 
during $P$. ginseng cultivation. The microbial community can be used as a sensitive biological indicator of soil health and function [15] and associates well with root disease suppression [27]. In this study, the richness and diversity of bacterial communities in forest soils without $P$. ginseng plantation (LCK) were significantly higher than in farmland soils (NCK) (Fig. 2a), suggesting a better soil function in the deforestation field.

The developmental stage of a plant is an important driving force for microbial community structure [17]. The richness and diversity of microbial communities (including bacteria and fungi) in farmland were found to change significantly with the cropping years of $P$. ginseng (Fig. 2b). The diversity of bacteria in the farmland increased after $P$. ginseng planting, while the fungal diversity decreased significantly in the first year of $P$. ginseng planting, compared to that in NCK. However, the variation trend of bacterial richness and diversity in the forest soil after planting $P$. ginseng was similar to that of the farmland. It changed significantly in the first three years of planting $P$. ginseng, and gradually stabilized in the fourth and fifth years. The richness and diversity of fungi were less affected by the number of years of $P$. ginseng planting (Fig. S1B). During the first to third years, $P$. ginseng is in the seedling stage; at this stage, the soil microbial community changed significantly and showed an undulating trend. In the maturity stage (the fourth to sixth years), when $P$. ginseng is ready to be harvested, the soil microbial community tended to stabilize.

The soil samples of different ages of $P$. ginseng planting were obtained from different locations, but the pattern of microbial community composition was similar in the locations with the same planting pattern. However, the microbial community composition of soil samples from the two different planting modes was significantly different. The bacterial phyla Proteobacteria, Actinobacteria, Acidobacteria, and Chloroflexi, and the fungal phylum Ascomycota were the most abundant during $P$. ginseng cultivation, indicating their widespread presence in the soil ecological system of $P$. ginseng monoculture, which is consistent with previous reports on cotton [18], soybean [28], and peanut [29] under continuous planting regimes. In the bacterial communities, the ratio of Proteobacteria to Acidobacteria is an index of soil nutrients, in which Proteobacteria is associated with eutrophic soil, and Acidobacteria is associated with infertile soil [30, 31]. Proteobacteria plays a crucial role in the global carbon, nitrogen, sulfur, and iron cycles [32, 33], and was the most abundant (Fig. 5a), which is consistent with findings of previous studies [34-36]. Actinobacteria was the second most abundant; it participates in the global carbon cycling [37] and the decomposition of soil organic material [38]. In an earlier study, Actinobacteria was used as a bio-control agent to control plant diseases transmitted by soil and seeds [39]. Similar to our findings, the primary bacteria in $P$. ginseng soil were Proteobacteria and Actinobacteria [26]. Interestingly, the abundance of Actinomyces varied with the $P$. ginseng cultivation, indicating that the $P$. ginseng cropping remarkably influenced the community of Actinobacteria. Therefore, the members of both Proteobacteria and Actinobacteria have a potential role in the microbial homeostasis in the continuous cropping soil.

Soil traits and crop varieties can have impact on the rhizosphere microbial community [40, 41], and the cropping year is considered to be a key factor driving this [34].The study showed that the microbiological compositions of the $P$. ginseng rhizospheric soil varied markedly with cultivation years. This phenomenon may be caused by the root exudates during the different growth stages [4244], which can alter rhizospheric microbial community, during plant growth $[40,45]$. P. ginseng also contains allelochemicals that can disturb the balance of microbial community [46], and reduce beneficial fungi while increase pathogenic fungi in $P$. ginseng rhizospheric soil [47].

Mortierella was the most abundant genus in the P. ginseng rhizospheric soil samples. It is widespread in the temperate zone and has not been previously reported as a P. ginseng pathogen. Fusarium, the third mostenriched genus, is a pathogen of many plants. F. solani was found to be the primary pathogenic species causing P. ginseng root rot. F.solani accumulated with the growth of $P$. ginseng, especially in the forest field. Furthermore, Gibberella, a perfect stage for Fusarium [25], is also an important soil-borne pathogen. Other major P. ginseng soil-borne pathogens, Cylindrocarpon and Ilyonectria that cause ginseng rust rot disease, also varied in abundances with the cultivation of $P$. ginseng. Currently, the isolates of Ilyonectria species that infect $P$. ginseng are categorized into four species: I. robusta, I. mors-panacis, I. panacis, and $I$. crassa $[48,49]$. In this study, I. morspanacis and $I$. robusta were noticeably enriched in the soil with $P$. ginseng cultivation, and the extent of accumulation varied with different cultivation years and soil types. N. ramulariae (anamorph: C. obtusiusculum) is a well-known soil-born fungus across the world [50]. Root rot of ginseng induced by Fusarium, Cylindrocarpon, and Ilyonectria is the main reason for the declined survival rate of $P$. ginseng. This study revealed that the total concentration of pathogenic species of $P$. ginseng increased annually during the first to fourth years of $P$. ginseng planting, reached the maximum in the fourth year, and decreased significantly in the fifth year, in farmland as well as in the forest field (Fig. 6b). I. morspanacis was specifically enriched in the fourth year of $P$. ginseng growth in the farmland and the forest field, while I. robusta and N. ramulariae were specifically enriched in the forest field. M. cucumerinaisa fungal plant 
Table 1 A detailed description of soil sample collection and characteristics $(n=5)$

\begin{tabular}{|c|c|c|c|c|c|c|c|}
\hline \multirow{2}{*}{$\begin{array}{l}\text { Sample } \\
\text { name }\end{array}$} & \multirow{2}{*}{$\begin{array}{l}\text { Cultivation } \\
\text { mode }\end{array}$} & \multirow{2}{*}{$\begin{array}{l}\text { Cultivation } \\
\text { age }\end{array}$} & \multirow{2}{*}{$\begin{array}{l}\text { GPS } \\
\text { coordinates }\end{array}$} & \multicolumn{2}{|l|}{ Bacteria } & \multicolumn{2}{|l|}{ Fungi } \\
\hline & & & & Total reads & OTUs & Total reads & OTUs \\
\hline NCK & Farmland & 0 & $125.61 \mathrm{E}, 41.54 \mathrm{~N}$ & $16,235,173$ & 2643 & $11,657,696$ & 1255 \\
\hline N1 & & 1 & $125.61 \mathrm{E}, 41.54 \mathrm{~N}$ & $17,742,598$ & 2475 & $10,161,450$ & 297 \\
\hline N2 & & 2 & $125.60 \mathrm{E}, 41.54 \mathrm{~N}$ & $17,241,033$ & 2772 & $9,555,316$ & 776 \\
\hline N3 & & 3 & $125.61 \mathrm{E}, 41.54 \mathrm{~N}$ & $15,425,882$ & 2440 & $8,493,303$ & 728 \\
\hline N4 & & 4 & $125.94 \mathrm{E}, 40.98 \mathrm{~N}$ & $15,734,231$ & 2381 & $9,271,211$ & 496 \\
\hline N5 & & 5 & $125.94 \mathrm{E}, 40.98 \mathrm{~N}$ & $17,114,356$ & 2465 & $9,734,170$ & 491 \\
\hline LCK & Forest field & 0 & $125.80 \mathrm{E}, 41.49 \mathrm{~N}$ & $16,475,198$ & 2117 & $9,363,764$ & 881 \\
\hline L1 & & 1 & $125.80 \mathrm{E}, 41.49 \mathrm{~N}$ & $15,773,793$ & 2545 & $10,379,872$ & 1138 \\
\hline L2 & & 2 & $125.80 \mathrm{E}, 41.49 \mathrm{~N}$ & $16,727,217$ & 1962 & $10,761,135$ & 934 \\
\hline L3 & & 3 & $125.80 \mathrm{E}, 41.49 \mathrm{~N}$ & $17,050,744$ & 2478 & $10,665,062$ & 718 \\
\hline$\llcorner 4$ & & 4 & $125.79 \mathrm{E}, 41.48 \mathrm{~N}$ & $15,674,442$ & 2410 & $9,016,774$ & 1024 \\
\hline L5 & & 5 & $125.79 \mathrm{E}, 41.48 \mathrm{~N}$ & $16,904,119$ & 2385 & $9,948,435$ & 939 \\
\hline
\end{tabular}

pathogen, and was found to accumulate the most among the aforementioned five pathogenic species. Monographella increased in abundance in the rhizosphere soil of $P$. quinquefolius when root rot occurred $[51,52]$.

Overall, it is likely that the increase in the abundances of soil-borne pathogenic fungi and the decrease in the abundances of other non-pathogenic fungi occurred under continuous single-cropping of $P$. ginseng. When the soil microbial community is disordered and rich in pathogenic microorganisms, it becomes difficult to control root colonization and insurgence of fatal disease seven under appropriate conditions. This study offers new insights into the influences of continuous planting $P$. ginseng on rhizosphere soil microbial communities.

\section{Conclusions}

The bacterial and fungal taxa revealed a remarkable difference in composition among the two types of fields with continuous cropping of $P$. ginseng. These effects jointly lead to disturbed microbial communities, which consequently result in serious soil-borne diseases. The key reasons for intercropping $P$. ginseng were the imbalance of the rhizosphere microbial community and the increase in the abundances of soil-borne pathogens. The results of this study reveal the effects of $P$. ginseng succession cropping on the diversity and composition of soil microbial communities. The change in microbial community composition is accompanied by the change in microbial functional characteristics. The soil-borne pathogens were accumulated through cropping years of $P$. ginseng. Therefore, the transplantation seems valid to avoid succession cropping $P$. ginseng. This partly explains why $P$. ginseng planted on farmland is usually transplanted in the second or third year.

\section{Materials and methods}

\section{Collection of rhizosphere soil samples}

$P$. ginseng rhizosphere soil samples were collected in June 2017 from different Standardized Planting Base (See Table 1 for details of sample collection locations) using conventional methods. The soil samples were collected from the rhizosphere areas (N1/L1, N2/L2, N3/ L3, N4/L4, and N5/L5) of $P$. ginseng with continuous planting for one, two, three, four, and five years, respectively. The control soil samples (NCK and LCK) were collected from the farmland and the deforestation field respectively where $P$. ginseng had not been planted. Each planting age in this study represented a different sampling location. From each sampling location, an area of $100 \mathrm{~m}^{2}$ was selected. In this area, five individual samples of $P$. ginseng plants were collected by the five-point sampling method. Therefore, these five biological repetitions came from the same location. $P$. ginseng roots were removed from the soil, topsoil was shakenoff, and then the soil close to the root surface was gently scraped off using cotton swabs (at least $5 \mathrm{~g}$ ). The collected soil samples were placed in labeled sterile bags and then transported to the laboratory at low temperature. From the five samples at each sampling point, three samples were randomly taken for sequencing and further analyses.

DNA extraction, amplicon generation, and Illumina MiSeq sequencing

Microbial genomic DNA from $P$. ginseng rhizospheric soil samples was extracted using E.Z.N.A. ${ }^{\circledR}$ Soil DNA Kit(OMEGA, U.S.) following the operation manual. The concentration and purification of the extracted DNA was first determined by the NanoDrop 2000ultraviolet-visible spectrophotometer (Thermo Scientific, U.S.), and the quality was evaluated by electrophoresis on a $1 \%$ agarose gel. 
Amplicon generation. The specific primer set $338 \mathrm{~F}$ (5' - ACTCCTACGGGAGGCAGCAG-3') / 806R (5'GGACTACHVGGGTWTCTAAT-3') [53] and ITS1F (5'-CTTGGTCATTTAGAGGAAGTAA-3') / ITS2R (5'-GCTGCGTT CTTCATCGATGC-3') [54] with barcode sequence were used to PCR-amplify the V3-V4 regions of the bacterial16S rRNA genes (468 bp product), and the ITS regions of fungi (300 bp product), respectively. PCR was conducted on the ABI GeneAmp 9700 thermocycler PCR system.PCR reaction procedure was: denaturation at $95^{\circ} \mathrm{C}$ for $3 \mathrm{~min}$; then 27 cycles of denaturation at $95^{\circ} \mathrm{C}$ for $30 \mathrm{~s}$, annealing at $55^{\circ} \mathrm{C}$ for $30 \mathrm{~s}$, and extension at $72{ }^{\circ} \mathrm{C}$ for $45 \mathrm{~s}$; and a final extension at $72{ }^{\circ} \mathrm{C}$ for $10 \mathrm{~min}$. Twenty microliters of the reaction system were consisted of $5 \times$ FastPfu Buffer $(4 \mu \mathrm{L}), 2.5 \mathrm{mM}$ dNTPs $(2 \mu \mathrm{L}), 5 \mu \mathrm{M}$ single primer $(0.8 \mu \mathrm{L})$, FastPfu Polymerase $(0.4 \mu \mathrm{L})$,and $10 \mathrm{ng}$ of template DNA. Three repeated PCR products per sample were pooled and detected by $2 \%$ agarose gel electrophoresis. The DNA fragments in the gel were purified and quantified according to the instruction manual of the AxyPrep DNA Gel Extraction Kit (Axygen Biosciences, U.S.) and QuantiFluor $^{\text {rm }}$-ST (Promega, U.S.), respectively.

The purified amplicons were pooled in an equimolar ratio according to the standard procedures of Majorbio Bio-Pharm Technology Co., Ltd. (Shanghai, China), and paired-end sequenced on the Illumina MiSeq platform (TruSeq ${ }^{\text {Tw }}$ DNA SamplePrep Kit, Illumina, U.S.). The raw reads were deposited into the NCBI Sequence Read Archive (SRA) database (Accession Number: PRJNA681095 and PRJNA681348).

\section{Sequencedata processing}

The raw FastQ files were demultiplexed, quality filtered by Trimmomatic [55], and then merged by FLASH [56] according to the following criteria: (i)the reads were truncated at any site receiving an average quality score $<$ 20 over a 50 bp sliding window,(ii) primers were exactly matched allowing 2 nucleotide mismatch and reads containing ambiguous bases were removed, and (iii) sequences with longer than 10 bp overlap were merged according to their overlap sequence.

UPARSE(version 7.1, http://drive5.com/uparse/) [57] was used to cluster operational taxonomic units (OTUs) with $97 \%$ similarity cut off, and UCHIME (version 4.2 ) was used to identify and remove the chimeric sequences. The RDP Classifier (http://rdp.cme.msu.edu/) was used to analyze the taxonomy of each 16S rRNA gene and ITS sequences against the Silva (SSU132) 16S rRNA database and Unite (version 8.0) ITS database, respectively, using a confidence threshold of $70 \%$. Then, the data were analyzed using the free online Majorbio ISanger Cloud Platform (www.i-sanger.com).
The indices of alpha diversity were analyzed by Mothur (version1.30.2). Forthe beta diversity analysis, the Bray-Curtis distance algorithm was used to calculate the distance between samples. The principal coordinates (PCoA) analysis was tested by ANOSIM. The Permutational Multivariate Analysis of Variance (PERMANOVA) was performed using the Bray-Curtis distance algorithm with 999 permutations.

\section{Statistical analysis}

Alpha diversity metrics of different groups were compared by Student's t-test. For comparative analysis of multiple groups, one-way ANOVA was conducted. The Student's t-test was used to compare the two groups. Statistical significance was set at $" p<0.05$, ${ }^{* *} p<0.01$, and $* * * 0<0.001$.

\section{Supplementary Information}

The online version contains supplementary material available at https://doi. org/10.1186/s12866-020-02081-2.

\begin{abstract}
Additional file 1: Figure S1. Estimation of microbial communities by alpha diversity. A, Alpha diversity of fungal communities between NCK and LCK. B, Alpha diversity of fungal communities among LCK, L1, L2, L3, $L 4$, and $L 5$. Estimation of alpha diversity representing two biological replicates for the NCK samples, and three biological replicates for the each rhizospheric soil sample $\left({ }^{*} p<0.05,{ }^{* *} p<0.01,{ }^{* * *} p<0.001\right)$.

Additional file 2: Figure S2. Comparison of abundance of bacteria at the phylum level. Three biological replicates for each rhizosphere soil sample $\left({ }^{*} p<0.05,{ }^{* *} p<0.01,{ }^{* * *} p<0.001\right)$. Major contributing phyla are displayed in different colors.
\end{abstract}

Additional file 3: Figure S3. Comparison of bacterial abundance in the samples from two soil types at the phylum level. A, Significant differences with cultivation ages among farmland soil samples ( $N$ group). B, Significant differences with cultivation ages among forest filed soil samples ( $L$ group). Three biological replicates for each rhizospheric soil sample $\left({ }^{*} p<0.05,{ }^{* *} p<0.01,{ }^{* * *} p<0.001\right)$.

Additional file 4: Figure S4. Comparison of fungal abundance at the phylum level. Three biological replicates for each rhizosphere soil sample $\left({ }^{*} p<0.05,{ }^{* *} p<0.01,{ }^{* * *} p<0.001\right)$. Major contributing phyla are displayed in different colors.

Additional file 5: Figure S5. Comparison of fungal abundance in the two samples at the phylum level. Three biological replicates for each rhizosphere soil sample $\left({ }^{*} p<0.05,{ }^{* *} p<0.01,{ }^{* * *} p<0.001\right)$.

\section{Acknowledgments}

This work was supported by the Science Technology Development Plan Project of Jilin Province (20150101084JC and 20190304012YY), and the Science and Technology Project of Jilin Education Department (TJKH20170438KJ).

\section{Authors' contributions}

WL and JYZ conceived and designed the experiments. WL,AZT and QL performed the experiments. WL, AZT, GQX and JYZ performed the data analysis. WL, AZT, and GQX wrote the manuscript. All authors read and approved the final manuscript. All authors have read and agreed to the published version of the manuscript.

Competing interests

The authors declare that they have no competing interests. 


\section{Author details}

${ }^{1}$ School of Life Science, Tonghua Normal University, No.950 Yu Cai Road, Dongchang District, Tonghua 134002, China. ${ }^{2}$ Chinese Institute of Jilin Ginseng, Tonghua 134002, China.

\section{Received: 24 September 2020 Accepted: 22 December 2020} Published online: 08 January 2021

\section{References}

1. Liu P, Yin H, Xu Y, Zhang Z, Chen K, Li Y. Effects of ginsenoside Rg1 on postimplantation rat and mouse embryos cultured in vitro. Toxicol in Vitro. 2006;20(2):234-8.

2. Liu P, Xu YJ, Yin HJ, Wang JB, Chen KJ, LI Y. Developmental toxicity research of ginsenoside Rb1 using a whole mouse embryo culture model. Birth Defects Res Part B. 2005;74(2):207-9.

3. Ramarao P, Bhargava HN. Antagonism of the acute pharmacological actions of morphine by panax ginseng extract. Gen Pharmacol. 1990;21(6):877-80.

4. Yu J, Eto M, Akishita M, Kaneko A, Ouchi Y, Okabe T. Signaling pathway of nitric oxide production induced by ginsenoside $\mathrm{Rb} 1$ in human aortic endothelial cells: a possible involvement of androgen receptor. Biochem Biophys Res Commun. 2007;353(3):764-9.

5. Tsutsumi YM, Tsutsumi R, Mawatari K, Nakaya Y, Kinoshita M, Tanaka K, Oshita S. Compound K, a metabolite of ginsenosides, induces cardiac protection mediated nitric oxide via Akt/PI3K pathway. Life Sci. 2011;88(1516):725-9.

6. Luo P, Dong GT, Liu L. The Long-Term Consumption of Ginseng Extract Reduces the Susceptibility of Intermediate-Aged Hearts to Acute Ischemia Reperfusion Injury. PLoS One 2015;10(12):e0144733.

7. Sagar SM, Yance D, Wong RK. Natural health products that inhibit angiogenesis:a potential source for investigational new agents to treat cancer-part 1. Curr Oncol. 2006;13(1):14-26.

8. Sagar SM, Yance D, Wong RK. Natural health products that inhibit angiogenesis: a potential source for investigational new agents to treat cancer-part 2. Curr Oncol. 2006;13:99-107.

9. Wan QX, Hyung KC, Lin FH. State of Panax ginseng Research: A Global Analysis. Molecules. 2017;22(9):1518.

10. Jung JD, Park HW, Hahn Y, Hur CG, In DS, Chung HJ, Liu JR, Choi DW. Discovery of genes for ginsenoside biosynthesis by analysis of ginseng expressed sequence tags. Plant Cell Rep. 2003;22(3):224-30.

11. Kirk JL, Beaudette LA, Hart M, Moutoglis P, Klironomos JN, Lee H, Trevors JT. Methods of studying soil microbial diversity. J Microbiol Methods. 2004; 58(2):169-88.

12. Smith KP, Goodman RM. Host variation for interactions with beneficial plant-associated microbes. Annu Rev Phytopathol. 1999;37:473-91.

13. Yao H, He Z, Wilson MJ, Campbell CD. Microbial biomass and community structure in a sequence of soils with increasing fertility and changing land use. Microb Ecol. 2000;40(3):223-37.

14. Selosse MA, Baudoin E, Vandenkoornhuyse P. Symbiotic microorganisms, a key for ecological success and protection of plants. C R Biol. 2004;327(7): 639-48.

15. Zuppinger DD, Schmid B, Petermann JS, Yadav V, De Deyn GB, Flynn DF. Selection for niche differentiation in plant communities increases biodiversity effects. Nature. 2014;515(7525):108-11.

16. Dong $L L, X u J$, Guang QF, Xi WL, Shi LC. Soil bacterial and fungal community dynamics in relation to Panax notoginseng death rate in a continuous cropping system. Sci Rep. 2016;6:31802.

17. Houlden A, Timms-Wilson TM, Day MJ, Bailey MJ. Influence of plant developmental stage on microbial community structure and activity in the rhizosphere of three field crops. FEMS Microbiol Ecol. 2008:65(2):193-201.

18. Xi H, Shen J, Qu Z, Yang D, Liu S, Nie X, Zhu L. Effects of long-term cotton continuous cropping on soil microbiome. Sci Rep. 2019;9(1):18297.

19. Tan Y, Cui Y, Li H, Kuang A, Li X, Wei Y, Ji X. Rhizospheric soil and root endogenous fungal diversity and composition in response to continuous Panax notoginseng cropping practices. Microbiol Res. 2017;194:10-9.

20. Dong L, Xu J, Zhang L, Yang J, Liao B, Li X, Chen S. High-throughput sequencing technology reveals that continuous cropping of American ginseng results in changes in the microbial community in arable soil. Chin Med. 2017;12:18.

21. Ying $Y X$, Ding $W L$, Zhou YQ, Li Y. Influence of Panax ginseng continuous cropping on metabolic function of soil microbial communities. Chin Herbal Med. 2012;4(4):329-34
22. Li Y, Ying YX, Zhao DY, Ding WL. Microbial community diversity analysis of Panax ginseng rhizosphere and non-rhizosphere soil using randomly amplified polymorphic DNA method. Open J Genet. 2012;2(2):95-102.

23. Zhou XG, Wu FZ. Dynamics of the diversity of fungal and Fusarium communities during continuous cropping of cucumber in the greenhouse. FEMS Microbiol Ecol. 2012;80(2):469-78.

24. Xiao C, Yang L, Zhang L, Liu C, Han M. Effects of cultivation ages and modes on microbial diversity in the rhizosphere soil of Panax ginseng. J Ginseng Res. 2016;40(1):28-37.

25. Vigier B, Reid LM, Dwyer LM, Stewart DW, Sinha RC, Arnason JT, Butler G. Maize resistance to gibberella ear rot: symptoms, deoxynivalenol, and yield. Can J Plant Pathol. 2001;23(1):99-105.

26. Vendan R, Lee $S, Y u Y$, Rhee $Y$. Analysis of bacterial community in the ginseng soil using denaturing gradient gel electrophoresis (DGGE). Indian J Microbiol. 2012;52(2):286-8.

27. Mazzola M. Assessment and management of soil microbial community structure for disease suppression. Annu Rev Phytopathol. 2004:42:35-59.

28. Li CG, Li XM, Kong WD, Wu Y, Wang JG. Effect of monoculture soybean on soil microbial community in the Northeast China. Plant Soil. 2010;330(1-2): 423-33.

29. Li XG, Ding CF, Zhang TL, Wang XX. Fungal pathogen accumulation at the expense of plant-beneficial fungi as a consequence of consecutive peanut monoculturing. Soil Biol Biochem. 2014;72:11-8

30. Castro HF, Classen AT, Austin EE, Norby RJ, Schadt CW. Soil microbial community responses to multiple experimental climate change drivers. Appl Environ Microbiol. 2010;76(4):999-1007.

31. Smit E, Leeflang P, Gommans S, van den Broek J, van Mil S, Wernars K. Diversity and seasonal fluctuations of the dominant members of the bacterial soil community in a wheat field as determined by cultivation and molecular methods. Appl Environ Microbiol. 2001;67(5):2284-91.

32. Hedrich S, Schlomann M, Johnson DB. The iron-oxidizing proteobacteria. Microbiology. 2011;157(Pt 6):1551-64.

33. Rosenzweig N, Tiedje JM, Quensen JF, Meng QX, Hao JJ. Microbial communities associated with potato common ccab-suppressive soil determined by pyrosequencing analyses. Plant Dis. 2012;96(5):718-25.

34. She SY, Niu JJ, Zhang C, Xiao YH, Chen W, Dai LJ, Liu XD, Yin HQ. Significant relationship between soil bacterial community structure and incidence of bacterial wilt disease under continuous cropping system. Arch Microbiol. 2017;199(2):267-75.

35. Wu X, Zhao QY, Zhao J, Xun WB, Li R, Zhang RF, Wu HS, Shen QR. Different continuous cropping spans significantly affect microbial community membership and structure in a vanilla-grown soil as revealed by deep pyrosequencing. Microb Ecol. 2015;70(1):209-18.

36. Tian Y, Gao L. Bacterial diversity in the rhizosphere of cucumbers grown in soils covering a wide range of cucumber cropping histories and environmental conditions. Microb Ecol. 2014;68(4):794-806.

37. Fradin EF, Thomma BP. Physiology and molecular aspects of Verticillium wilt diseases caused by V. dahliae and V. albo-atrum. Mol Plant Pathol. 2006;7(2):71-86.

38. Rima U, Chih YC, Karin E, Glen D, David CC, William BW. Differences in the composition and diversity of bacterial communities from agricultural and forest soils. Soil Biol Biochem. 2008;40(6):1294-305.

39. Palanisamy $P$, Dharmadurai D. Diversity of soil Allelopathic Actinobacteria in Tiruchirappalli district, Tamilnadu, India. J Saudi Soc Agric Sci. 2015;14(1):54-60.

40. Berg G, Smalla K. Plant species and soil type cooperatively shape the structure and function of microbial communities in the rhizosphere. FEMS Microbiol Ecol. 2009;68(1):1-13.

41. Micallef SA, Shiaris MP, Colon-Carmona A. Influence of Arabidopsis thaliana accessions on rhizobacterial communities and natural variation in root exudates. J Exp Bot. 2009;60(6):1729-42.

42. Chaparro JM, Badri DV, Bakker MG, Sugiyama A, Manter DK, Vivanco JM. Root exudation of phytochemicals in Arabidopsis follows specific patterns that are developmentally programmed and correlate with soil microbial functions. PLoS One. 2013;8(2):e55731.

43. Yu JQ, Matsui Y. Phytotoxic substances in root exudates of cucumber (Cucumis sativus L.). J Chem Ecol. 1994;20(1):21-31.

44. Dong L, Xu J, Zhang L, Cheng R, Wei G, Su H, Yang J, Qian J, Xu R, Chen S. Rhizospheric microbial communities are driven by Panax ginseng at different growth stages and biocontrol bacteria alleviates replanting mortality. Acta Pharm Sin B. 2018;8(2):272-82. 
45. Chaparro JM, Badri DV, Vivanco JM. Rhizosphere microbiome assemblage is affected by plant development. ISME J. 2014;8(4):790-803.

46. Wu L, Wang J, Huang W, Wu H, Chen J, Yang Y, Zhang Z, Lin W. Corrigendum: plant-microbe rhizosphere interactions mediated by Rehmannia glutinosa root exudates under consecutive monoculture. Sci Rep. 2016;6:19101.

47. Li Z, Fu J, Zhou R, Wang D. Effects of phenolic acids from ginseng rhizosphere on soil fungi structure, richness and diversity in consecutive monoculturing of ginseng. Saudi J Biol Sci. 2018;25(8):1788-94.

48. Cabral A, Groenewald JZ, Rego C. Cylindrocarpon root rot: Multi-gene analysis reveals novel species within the llyonectria radicicola species complex. Mycol Prog. 2012;11(3):655-88.

49. Guan Y, Chen M, Ma Y, Du Z, Yuan N, Li Y, Xiao J, Zhang Y. Whole-genome and time-course dual RNA-Seq analyses reveal chronic pathogenicity-related gene dynamics in the ginseng rusty root rot pathogen llyonectria robusta. Sci Rep. 2020;10(1):1586.

50. Hirooka YI. Yu;Masuya, Hayato;Kubono, Takanori. Seed rot, a new disease of beech tree caused by Neonectria ramulariae (anamorph: Cylindrocarpon obtusiusculum). J Phytopathol. 2012;160(9):504-6.

51. Yu M, Jiang JL, Ren XM, Li L, Jiao CJ, Yang L, Xu H. Research on relationship between occurrence of root rot and changes of fungal communities in rhizosphere of Panax quinquefolius. Zhongguo Zhong Yao Za Zhi. 2018; 43(10):2038-47.

52. Du J, Qv M, Qv W, Liu L, Zhang Y, Cui M, Zhang H. Potential threats of zeolitic imidazolate framework-8 nanoparticles to aquatic fungi associated with leaf decomposition. J Hazard Mater. 2021:401:123273.

53. Xu NT, Guangcai, Wang H. Effect of biochar additions to soil on nitrogen leaching, microbial biomass and bacterial community structure. Eur I Soil Biol. 2016;74:1-8

54. Rachel IA, Marzia M, John WT, Thomas DB. Dispersal in microbes: fungi in indoor air are dominated by outdoor air and show dispersal limitation at short distances. ISME J. 2013;7:1262-73.

55. Bolger AM, Lohse M, Usadel B. Trimmomatic: a flexible trimmer for Illumina sequence data. Bioinformatics. 2014;30(15):2114-20.

56. Magoc T, Salzberg SL. FLASH: fast length adjustment of short reads to improve genome assemblies. Bioinformatics. 2011;27(21):2957-63.

57. Edgar RC. UPARSE: highly accurate OTU sequences from microbial amplicon reads. Nat Methods. 2013;10(10):996-8.

\section{Publisher's Note}

Springer Nature remains neutral with regard to jurisdictional claims in published maps and institutional affiliations.

Ready to submit your research? Choose BMC and benefit from:

- fast, convenient online submission

- thorough peer review by experienced researchers in your field

- rapid publication on acceptance

- support for research data, including large and complex data types

- gold Open Access which fosters wider collaboration and increased citations

- maximum visibility for your research: over $100 \mathrm{M}$ website views per year

At $\mathrm{BMC}$, research is always in progress.

Learn more biomedcentral.com/submissions 